\title{
Bitte wenden Sie jetzt - Das Auto der Zukunft von Eckard Helmers
}

\author{
WILEY-VCH Verlag GmbH \& Co. KGaA, Weinheim. Oktober 2009. ISBN: 978-3-527-32648-8. \\ 204 Seiten, gebunden. $€ 24,90$. http://www.wiley-vch-macht-neugierig.de/02_helmers/buch/ \\ bitte_wenden.php
}

\begin{abstract}
Almut B. Heinrich
Online veröffentlicht: 24. November 2009

(C) Springer-Verlag 2009
\end{abstract}

- Darstellung und Bewertung klassischer und alternativer Antriebe für PKW hinsichtlich ihrer Umwelt- und Gesundheitswirkungen (externe Folgekosten),

- Beurteilung des Umwelt- und Gesundheitseffektes des europäischen Diesel-PKW-Booms, zum Beispiel vor dem Hintergrund aktueller Klimawirkungsdaten von Ruß,

- Chancen und Grenzen von Erdgas- und Flüssiggasantrieben,

- Wirkungsgrade alternativer, auch biogener Treibstoffe,

- Antriebseffizienzen im Vergleich (WTW, well to wheel), z.B. von Brennstoffzellenfahrzeugen und

- Umwelt- und Gesundheitswirkungen des Einsatzes von Elektroautos. Hierzu sind erstmals auch die indirekten Stickoxid-, Feinstaub- und Schwefeldioxidemissionen berechnet worden, die über die Stromerzeugung fällig werden.

Darüber hinaus wird der Frage nachgegangen, warum es so schwer ist, die Autoindustrie zur Serienfertigung neuer Technikkonzepte zu bewegen und unter welchen Umständen solche Innovationen in den vergangenen 100 Jahren in den Markt gelangten (z.B. ,technology forcing“ in den USA).

\section{Inhalt}

1 Über 100 Jahre Automobil - Woher kommen wir, wohin fahren wir? 1

Die Welt macht mobil 1

Automobilität in Zahlen 2

Automobilität aktuell 3

An der Ressourcengrenze: Die letzte Warnung? 5

Verdopplung von Gewicht und Leistung 8

Wie technische Evolution im Automobilsektor funktioniert 13 
Die Innovationsgeschichte des Automobils 16

Struktur des US-amerikanischen Emissions-Rechts 21

Innovationsfördernd: die US-amerikanische Umwelt-

politik 22

Der Widerstand gegen den Abgas-Katalysator 23

Die Nullemissions-Politik Kaliforniens 24

Deutschland und Europa 26

Die neuen CO2-Zielwerte für Europa 27

Staatsverflechtung 28

Auto- und Ölindustrie 31

2 Verbrennung produziert nicht nur CO2 - Die Schad-

stoffe und ihre Wirkungen 35

Die wichtigsten Luftschadstoffe und ihre Quellen 35

Wirkungen und Folgen der Luftschadstoffe 39

Stickoxide 40

Ozon 42

Feinstaub 45

Je kleiner, desto gefährlicher 47

Die Rolle des Autos als Verursacher von Luftverschmut-

zung 50

Globale Perspektive und Geschichte der Auto-Emissio-

nen 52

Ist Luftverschmutzung unausweichlich? Eine Bilanz 55

3 Was bedeutet Nachhaltigkeit beim Automobil? Eine

Bilanz des Dieselbooms 59

Der Treibhauseffekt und seine Verursacher 60

Klimawirksamkeit von Auto-Emissionen 62

Zusätzlicher Strahlungsantrieb durch Ruß und US-ameri-

kanische Simulation zum Effekt eines Dieselbooms 66

Der Dieselboom in Europa 71

Klimabilanz des Dieselbooms 73

Was steckt noch im Abgas? 76

Entstickung der Dieselabgase 82

Nachhaltigkeit von Antriebstechniken - quantitativ messbar? 84

\section{Die Alternativen 91}

Konventionelle Treibstoffe 93

Synthetische Treibstoffe 94

„Gas to liquid“ 95

BTL - „biomass to liquid“ 95

Regenerativ 96

Biokraftstoffe 97

Pflanzenöl 99

Alternative Bioethanol 102

Ökobilanzen 103

Nachhaltigkeit alternativer Antriebe 109

Herausragend: Elektromobilität 112

Wasserstoff und die Brennstoffzelle 113

Kraftstoff- und Antriebseffizienz in der Praxis 115

\section{Alternative heute - Gas geben 119}

Lufthygienisch relevante Emissionen der Gasverbren-

nung 121

Gasbetriebene Busse 121
Weitere klimarelevante Emissionen der Gasverbren-

nung 123

Berechnung der CO2-Emissonen bei der Gasverbrennung 125

Verbreitung der Mobilität mit Flüssiggas und Erdgas 125

Erdgas und Flüssiggas als alternative Kraftstoffe in

Deutschland 128

Praktikabilität von Erdgas- und Flüssiggasautos 129

Erdgasautos in der Praxis: Optimierungen 130

LPG-Autos in der Praxis: der Mehrverbrauch 132

LPG-Autos in der Praxis: Optimierungen 133

Welches Auto ist heute das ökologisch effizienteste? 135

6 Elektromobilität - Alternative von morgen 137

1940-1990: Übergangszeit 139

Nach 1996: Renaissance 140

Elektrohybridisierung 140

Energiespeicher für Elektroautos 144

Verbrauchsmessung 148

Verbrauchsangaben im Mischbetrieb elektrisch/verbrennungsmotorisch 149

Serieller Hybrid GM Volt/Opel Ampera 150

Zukünftige Akzeptanz von Elektroautos 152

Vereinfachung des Autobaus durch Elektromobile 156

Radnabenantrieb 158

Wie nachhaltig ist Elektromobilität? Die Sache mit

der CO2-Emission 160

CO2-Emissionen durch Elektromobilität (Stromerzeu-

gung) 161

Weitere Emissionen durch die Stromerzeugung 165

Externe Folgekosten durch Elektromobilität 167

Elektromobilität: Sauber bereits mit dem Strommix von

heute 169

Zukunftsvisionen 171

Vision für Europa 172

7 Nachhaltige Automobilität - Es geht um Technik, aber nicht nur 175

Ursachen des Dieselbooms 176

Spritspar-Sondermodelle 178

Anstieg der Mobilitätskosten 180

Klimafolgen der Dienstwagensubvention 181

Reale Emissionen in der Hochgeschwindigkeitskultur 182

Nachhaltige Mobilität: Technik ist nicht alles 185

Auto-Umweltlisten 186

Index

\section{Einleitung}

„Bitte wenden Sie jetzt“ klingt als Aufforderung aus den Navigationsgeräten, wenn wir in die falsche Richtung fahren. Automobiltechnik fährt im Prinzip seit 100 Jahren in die gleiche Richtung. Doch diese Straße wird immer schmaler. Immerhin hat sie Kreuzungen und Abzweigungen. Alterna- 
tiven liegen am Wegesrand bereits hinter uns: Autos fuhren vor langer Zeit bereits mit Strom. Die hier vorgestellte Situationsanalyse hinsichtlich Ressourcen, Alternativen, Umwelt- und Gesundheitskosten mündet in die Empfehlung: „Bitte wenden Sie jetzt“.

Dieses Buch ist aus der Sicht eines Umweltanalytikers geschrieben, der sich nicht für bestimmte Techniken begeistert, sondern sich für Wirkungen auf Mensch und Umwelt interessiert, weil er gesehen hat, wie Spuren- und Schadstoffe unsichtbar um die Welt reisen. Menschen haben ein fundamentales Recht auf körperliche Unversehrtheit. Wodurch diese aber wirklich beeinträchtigt wird, ist in den modernen Industriegesellschaften manchmal wenig bekannt. Wer weiß in Europa schon, dass die Luftqualität in europäischen Ballungszentren in weltweitem Vergleich zu wünschen übrig lässt und dass der Feinstaub eines der größten Lebensrisiken für einen Europäer darstellt?

Das nationalstaatliche und europäische institutionelle Handeln ist geprägt von dem, was öffentlich thematisiert wird. Dies erklärt zum Teil, warum europäische Politiker sich derzeit lieber mit $\mathrm{CO} 2$ befassen als mit den Luftschadstoffen, die vor Ort wirken. Anders formuliert: Hier geht es um die berühmte „Nachhaltigkeit“. Nachhaltigkeit bedeutet: Eine Technik hat nicht nur wenig Ressourcen zu verbrauchen, sondern vor allem Mensch und Umwelt möglichst wenig zu belasten. Es geht um die Auswirkungen auf die heutige und auf folgende Generationen. Hier gibt es großen Handlungsbedarf, denn Autos werden seit über 100 Jahren mit Verbrennungsmotoren betrieben, deren Emissionen Mensch und Umwelt viel abverlangen und hohe Kosten verursachen. Die Folgen allerdings, wie eine insgesamt deutlich verringerte Lebenserwartung, spielen sich im privaten Rahmen ab. Sie stehen deshalb meist abseits der öffentlichen Wahrnehmung.

Über hundert Jahre haben Autos unzählige Menschen bewegt, begeistert, ihre Produktion hat Millionen ernährt. Gleichzeitig wurden auch Millionen Menschen verletzt oder erkrankten. Hier soll jedoch nicht Automobilität in Frage gestellt, sondern im Gegenteil untersucht werden, wie sie in Zukunft mit deutlich weniger Kosten an Umwelt und Gesundheit betrieben werden kann.

Im pragmatischen Sinne ist dies nicht ein Buch über neue Technikideen. Es wird immer schon an neuen Antriebstechniken geforscht. Dennoch werden hier Hoffnungen wie die Gasturbine, der druckluftunterstützte Motor oder der Stirlingmotor nicht thematisiert. Am Brennstoffzellenfahrzeug wird seit Jahrzehnten geforscht, serienreif ist es immer noch nicht. Es ergibt keinen Sinn, weiter auf neue Techniken zu warten.

Der Ölpreisschock des Jahres 2008 und die Klimadiskussion haben deutlich gemacht, dass unser Mobilitätssystem „an die Wand zu fahren“ droht. Dieses Buch fragt deshalb: Was steht heute zum Umsteuern zur Verfügung? Vor allem:
Welche ist von den heute verfügbaren und den unmittelbar zukünftigen Optionen „,nachhaltiger“, also mit weniger Kosten an Umwelt und Gesundheit behaftet?

Die meisten sogenannten ,alternativen“ Treibstoffe und Antriebsstrategien, wie zum Beispiel der Elektrohybrid, wurden bereits vor 100 oder mehr Jahren entwickelt oder sogar eingesetzt. Dennoch werden seit dieser Zeit im Prinzip immer die gleichen Autos mit Verbrennungsmotor gebaut. Warum haben es Innovationen auf diesem Markt so schwer? Unter welchen Bedingungen hat es in der über 100-jährigen Geschichte der Massenmobilität echte Neuerungen am Markt gegeben? Grundlegende Innovationen waren sehr selten und deswegen ist es umso wichtiger, sich die Umstände anzusehen, unter denen sie auf den Markt gelangten. Die reale Technikentwicklung der vergangenen 100 Jahre kontrastiert völlig mit der Selbstdarstellung der Automobilbranche, die auf Messen hunderte von Konzeptautos vorstellt, aber ihre Produkte kaum jemals wirklich verändert hat.

Die entscheidende Frage ist vielleicht nicht, welche neue Technik uns rettet. Stattdessen sollte gefragt werden, warum sich über so lange Zeit so wenig änderte. Dies hat Gründe, die aufzuzeigen sind. Seit etwa 1990 steht die Elektromobilität in den Startlöchern, regional immer mal wieder reaktiviert. Seit wenigen Jahren wissen wir, dass ein erheblicher, wahrscheinlich der überwiegende Teil des Stroms selbst in gemäßigten Breitengraden regenerativ erzeugt werden kann. Dies erscheint heute als die realistische Lösung vieler Probleme. Elektromobilität und regenerative Stromerzeugung passen wohl auch vom Management her zueinander. Doch soll man weitere 20 bis 30 Jahre warten, bis Strom überwiegend regenerativ produziert wird? Die Vor- und Nachteile und die Umweltwirkungen von Elektromobilität gegenüber Autos mit konventionellen Verbrennungsmotoren werden hier deshalb auf gegenwärtiger Basis betrachtet. Mit welchen Emissionen ist zu rechnen, wenn Strom für Elektroautos mit dem heutigen Strommix erzeugt wird? Im Buch werden neue Berechnungen vorgestellt.

In diesem Zusammenhang muss wieder umfassender über die Schäden an Umwelt und Gesundheit geredet werden, die Emissionen von PKW mit Benzin- und Dieselmotor mit sich bringen. Die an sich berechtigte Klimadebatte hat diese Kosten aus dem Blickfeld wandern lassen. Mehr Nachhaltigkeit wird nicht erreicht, wenn sich die Umweltqualität bei einzelnen Schadstoffen verbessert, bei anderen jedoch verschlechtert. Doch genau dies bewirkte die CO2fixierte EU-Autopolitik seit Mitte der 1990er-Jahre, wie in diesem Buch begründet wird. In der CO2-Diskussion geht es im Kern um die Bedrohung durch eine globale Klimaerwärmung. Ist denn die EU-Autopolitik wenigstens in klimatischem Sinne nachhaltig, also wirksam?

In den folgenden Kapiteln wird die Klimawirkung des staatlich veranlassten Diesel-PKW-Booms in Europa seit 
den 1990er-Jahren diskutiert. Dieselautos bedeuten zwar keine grundsätzliche technische Revolution, dennoch ist der Technikwandel, der sich hier in Europa vollzogen hat, bemerkenswert. Wandel scheint also möglich. Es ist wichtig, hieraus für die Zukunft zu lernen: Was hat uns dieser Trend gebracht, wie ist es dazu gekommen und was können wir verbessern?

Biogene Treibstoffe erfreuen sich seit Jahren großen Interesses und gelten allein aus strategischen Gründen bisweilen als Retter in der Not. Werden Verbrennungsmotoren mit biogenen Treibstoffen betrieben, stellt das jedoch keine Grundinnovation dar und schafft auch nicht das Problem toxischer Emissionen auf den Straßen ab. Deshalb stehen die ,agrofuels“, wie sie von Kritikern genannt werden, auch nicht im Zentrum dieses Buches, sie sind aber ein wichtiger Baustein für zukünftige Mobilität. Treibstoffe aus nachwachsender Biomasse können die Zeit des ,phasing out" (langsamen Abschieds) vom fossilen Kohlenstoff in den nächsten 20-30 Jahren begleiten. Bis hauptsächlich Fahrzeuge mit innovativen Antrieben verkehren, werden noch Jahrzehnte vergehen. Heutige Kraftfahrzeuge mit Diesel- und Benzinmotoren können dagegen mit begrenztem Aufwand auf verschiedene biogene Treibstoffe umgerüstet werden. Auch wenn sich Elektromobilität zunächst im Regionalverkehr durchsetzt, fahren noch Millionen Nutzfahrzeuge über weite Strecken und sind dabei vielleicht weiterhin auf kohlenstoffbasierte Treibstoffe angewiesen. Hier können, soweit sinnvoll und mit Augenmaß, Biotreibstoffe eingesetzt werden.

Im Jahr 2008 schien ein Wendepunkt erreicht, wohl weniger durch Einsicht als durch den Ölpreisschock. Inzwischen sind die Preise wieder in sich zusammengefallen. Höchstwahrscheinlich handelt es sich jedoch nur um eine vorübergehende Periode gesunkener Ölpreise. Sie sollte nicht zu weiterem Abwarten führen.

Lösungen für Umweltprobleme setzen seit Jahrzehnten gerne „end of the pipe“, also am Ende der Leitung an. „End of the pipe" bedeutet: Kurz bevor Emissionen in die Umwelt entlassen werden, wird ein Katalysator oder Filter ergänzt, und dann noch einer und noch einer. In diesem Buch wird gezeigt, welch komplexes Chemielabor Diesel-PKW in Zukunft mit sich führen müssen, um die Emissionen auch nur auf das Niveau von Benzinern zu senken. Solche Lösungen sind letztlich nicht nachhaltig. Sie erfordern große Material- und Geldressourcen, arbeiten an den Symptomen, verhindern eine echte Lösung, die heißen muss: Erst gar keine Emissionen entstehen zu lassen, zumindest nicht auf den Straßen.

Emissionen aus der Verbrennung fossiler Rohstoffe sollten nach Möglichkeit nicht mehr unmittelbar in die Atmungsorgane von Menschen entlassen werden. Symptomatisch für den begrenzten Stellenwert der menschlichen Gesundheit in Europa ist es, dass die Abgasrohre von
Bussen und LKW in Kopfhöhe von Kleinkindern münden. Erwachsene liegen vielleicht eineinhalb Meter darüber. Im Vergleich dazu schaue man sich die Lösungen in den USA an. Jeden Tag stehen in Deutschland Schulkinder an Bushaltestellen, wo sie in schwarze Rauchwolken gehüllt werden. Viele Kommunen vergeben diese Transportaufgaben an den günstigsten Anbieter, und das ist nicht einer, der sich einen neuen Erdgas- oder Hybridbus leisten kann.

Verbrennung fossiler Energieträger sollte, wenn nötig, dort stattfinden, wo man die Abgase mit größter Sicherheit beseitigen kann, also in den Kraftwerken. Dies funktioniert effizienter und auch zuverlässiger als bei den Katalysatoren und Partikelfiltern der Kraftfahrzeuge, die natürlich beim heutigen Stand unverzichtbar sind. Wenn Kraftwerke Strom für Mobilität produzieren, dann nach Möglichkeit mit immer weniger fossilen Ressourcen und immer effizienter, schon aus strategischen Gründen.

Den Lesern des Buches soll auch nicht vorenthalten werden, dass man nicht alles weiß. So ist über die Chemie der Ozonentstehung - gemeint ist das für Mensch und Umwelt toxische, klimaerwärmende, bodennahe Ozon - viel geforscht und geschrieben worden. Welche Komponenten individuell vor Ort für die Zunahme von Ozon verantwortlich sind, ist jedoch umstritten. Allein die Messungen sind unbestreitbar - und die Werte gehen mancherorts nach oben.

Wo es viele Informationen gibt, kann auch leicht manipuliert werden. Wenn es um die Umweltwirkungen von Autoemissionen geht, greift so manche Interessengruppe nach dem, was passt. Wie leicht das geht, wird in diesem Buch am Beispiel des Feinstaubs aufgezeigt. Je nach gewünschter Aussage kann der Verkehr eine untergeordnete Quelle oder aber dominierend sein. Schadstoffe und ihre Wirkungen und Wechselwirkungen auf Mensch und Umwelt sind höchst komplex. Ein vermeintlich einfaches Thema wie $\mathrm{CO} 2$ kann da öffentlich noch vergleichsweise leicht vermittelt werden. Doch in Wirklichkeit geht es ja nicht (nur) um CO2, sondern um die Klimaerwärmung. Hier spielt Ruß ebenfalls eine zentrale Rolle, doch der wurde über Jahre vernachlässigt. Die Folge war eine nicht nachhaltige Umweltpolitik. Wo so viel Information, aber auch Desinformation ist, kann nur Selbstinformation helfen. Dazu möge dieses Buch einen Beitrag leisten. Die Informationsvielfalt ist manchmal eine Zumutung. Alles andere könnte jedoch unzulässige Vereinfachung sein.

Letztlich verändern sich Prioritäten in der Umweltpolitik und somit in der Luftreinhaltung nur durch fortgesetzte Kommunikation und öffentliche Auseinandersetzungen. Nachhaltige technische Innovationen werden oft nur auf öffentlichen Druck hin realisiert, wobei der Begriff der Nachhaltigkeit ebenfalls einem Wandel unterworfen ist. Dieses Buch soll daran mitwirken. Es ist wichtig, dass sich möglichst viele an der Diskussion beteiligen, um ein Gegengewicht zu den Verhandlungen zwischen Autoindustrie und 
EU-Kommission hinter verschlossenen Türen in Brüssel zu schaffen.

Es kann nicht nur um Arbeitsplätze gehen, sondern um eine Umwelt, in der man in körperlicher Unversehrtheit arbeiten und leben kann. Wirklich innovative Automobile stellen beides sicher. Eigentlich können nur Autos, die nicht mehr lärmen und stinken, bewirken, dass Menschen auf der ganzen Welt durchschnittlich einige Monate länger leben und dass solche Autos langfristig erfolgreich verkauft werden können.

Zum Schluss noch ein Hinweis an die Leserin und den Leser: Es geht hier am allerwenigsten darum, irgendjemandes Auto zu kritisieren. In diesem Buch werden etliche problematische Details über Dieselemissionen zusammengetragen. Wegen der wirtschaftlichen Dominanz der Autoindustrie werden solche Informationen nur selten genannt, ihre Darstellung ist deshalb umso wichtiger. Die Autoindustrie hat es geschafft, dass sich viele Kunden persönlich mit ihrem jeweiligen Auto identifizieren. Das ist das legitime Ziel industrieller Hersteller. Erreicht wird es durch Werbung und die Mitarbeit vieler Akteure, die im Umfeld der Industrie tätig sind. Autos sind in der Werbung und in verbreiteter öffentlicher Wahrnehmung sauber, effizient, modern, sportlich, also eigentlich in allem richtig. Die persönliche Identifikation vieler Menschen mit einem Produkt wie dem Auto macht den Wandel aber auch schwierig: „Es kann doch nicht sein, dass ich die Umwelt belaste, vielleicht mehr als andere ..." Auch der Autor belastet Umwelt und Mitmenschen durch Autofahren, soviel sei klargestellt.

Wenn gegenwärtig in manchen Staaten Mitteleuropas die meisten Menschen ein Dieselfahrzeug kaufen, dann ist das nur oberflächlich betrachtet eine private Konsumentenentscheidung. In Wirklichkeit handelt es sich um eine logische Folge staatlicher Lenkungspolitik. Die gesetzlichen Grenzwerte erlauben Diesel-PKW immer noch höhere Emissionen als Benzin-PKW, damit die Autoindustrie diese Produkte leichter vermarkten kann. Dieselautos dürfen mehr Stickoxide emittieren und sind deshalb preislich konkurrenzfähig. Der Staat zeichnet die Kaufentscheidungen der Kunden zusätzlich vor, in Deutschland aktuell mit der reformierten Kraftfahrzeugsteuer und seit langem ebenfalls in Deutschland mit der um rund $18 \mathrm{Cent} / \mathrm{L}$ niedrigeren Mineralölsteuer auf Dieseltreibstoff. Wenn ein Dieselauto im Betrieb billiger ist und die Industrie immer schon sagt, sie habe alle Umweltaspekte vorausschauend berücksichtigt, welchen Zweifel sollte es dann für den Kunden geben? Doch in der Realität stellen sich die Dinge anders dar, wie in diesem Buch vielfältig dargestellt.

Eine Rezension des Werkes erscheint in der Februarausgabe 2010 von „Umweltwissenschaften und SchadstoffForschung“".

Eckard Helmers ist seit 1998 Professor für Umweltanalytik am neu gegründeten Umweltcampus Birkenfeld. Zuvor war er Abteilungsleiter im Stuttgarter Umweltamt und hat auch einige Jahre in der Meeresforschung gearbeitet. Der Chemiker Eckard Helmers veröffentlichte 35 Fachartikel über Schwermetalle und Spurenstoffe in der Umwelt. Er ist seit 10 Jahren Mitherausgeber der Zeitschrift „Umweltwissenschaften und Schadstoff-Forschung“. Am Umweltcampus Birkenfeld engagiert er sich besonders in der Internationalisierung, zuletzt in einem umfangreichen EU-US-Projekt im Bereich „Biotechnology and Healthcare". 